\title{
Der Telenotarzt als Innovation des Rettungswesens im ländlichen Raum - eine gesundheitsökonomische Analyse für den Kreis Vorpommern-Greifswald
}
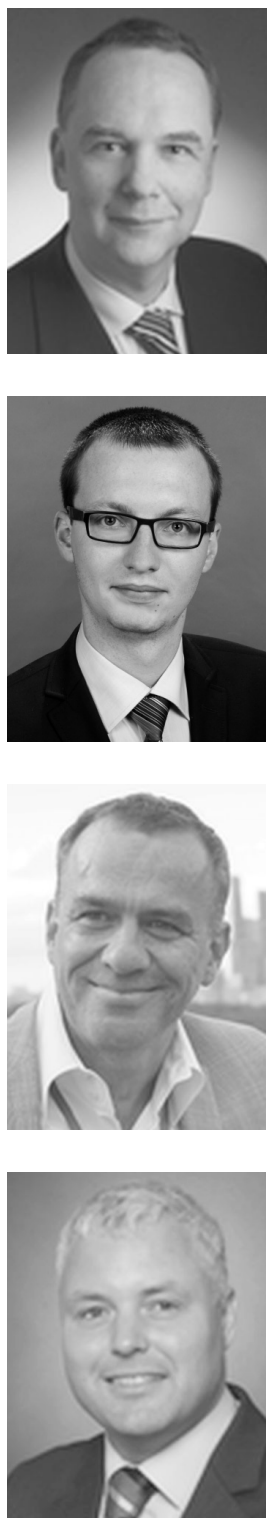

Steffen Fleßa, Markus Krohn, Dirk Scheer und Klaus Hahnenkamp

Break-Even-Analyse, Kostenvergleich, Notarztstandorte, Rettungswesen, Telenotarzt

Break-Even-Analysis, Cost Analysis, Emergency Doctor Position, Emergency Care, Telemedicine Emergency Doctor

Die notärztliche Versorgung in ländlichen Regionen steht vor der Herausforderung, einerseits die rechtzeitige Rettung sicherzustellen, andererseits keine unterausgelasteten Kapazitäten zu schaffen. Als Alternative zum Ausbau der Notarztstandorte bieten sich die Luftrettung und der so genannte „Telenotarzt“ an. Gerade in Regionen mit dünner Besiedelung und geografischen Barrieren wie in der Schweiz muss die Alternative gewählt werden, die die Einhaltung der Hilfsfrist gewährleistet und die Kosten minimiert. Die vorliegende Analyse vergleicht die Kosten der drei Varianten. Es zeigt sich, dass der Telenotarzt eine vielversprechende Innovation ist, die auch in der Schweiz in Zukunft noch relevanter sein dürfte.

The provision of emergency doctors in rural regions is challenged by guaranteeing early rescue while limiting the number of emergency doctor positions. The first alternative to achieve these objectives is the increase of the number of emergency medical services. Alternatives are helicopter rescue and the so-called "telemedicine emergency doctor". In particular in regions with low population density and geographical barriers (such as Switzerland) one has to choose an alternative which minimises the costs while still warranting that the emergency time is kept. This analysis compares the costs of all three alternatives. It becomes obvious that the telemedicine doctor is a promising innovation which is due to become more relevant for Switzerland. 


\section{Einführung}

Die Versorgung im Raum impliziert einen Konflikt zwischen Sicherheit und Effizienz. Aus Gründen der Versorgungssicherheit wäre eine flächendeckende, möglichst dichte Positionierung der Einrichtungen der Daseinsvorsorge sinnvoll, da dadurch die Zugänglichkeit erhöht bzw. die Anfahrtszeit minimiert wird (Dünkel, Herbst et al. 2014, Herbst, Dünkel et al. 2016). Aus Effizienzgründen sollten jedoch möglichst wenige Standorte vorgehalten werden, da - insbesondere bei fixkostenintensiven Betrieben - viele kleine Standorte höhere Fallkosten implizieren (Fleßa 2016). Insbesondere für Krankenhäuser im ländlichen Raum ist der Konflikt zwischen wohnortnaher und kostengünstiger Versorgung beschrieben (Fleßa und Gieseler 2016).

Dieser generelle Zielkonflikt tritt besonders eindrücklich in der notärztlichen Versorgung auf. Einerseits müssen alle möglichen Orte, an denen sich ein Notfall ereignen kann, möglichst schnell erreichbar sein, da eine verzögerte Rettung regelmäßig das Leben von Menschen gefährdet. Andererseits impliziert eine dichte, flächendeckende Versorgung in dünn besiedelten Gebieten oder in Regionen mit natürlichen Barrieren (Flüsse, Seen, Berge), dass viele Notarztwachen gebaut und betrieben werden müssen, die bei der geringen Bevölkerungsdichte nur unzureichend ausgelastet sind (Fleßa 2016). Die Schweiz ist hierfür ein sehr gutes Beispiel, da die Bevölkerungsdichte zwischen den Regionen stark schwankt und natürliche Hindernisse wie z. B. Täler, Berge und Seen die Zugänglichkeit einschränken. Hinzu kommt, dass Teile der Schweiz im Winter unzugänglich sind und die Nachfrage durch Touristen stark schwankt. Eine schnelle und gleichzeitig kostengünstige Rettung stellt eine schwierige Planungsaufgabe dar.

Die meisten Länder haben so genannte „Hilfsfristen“ definiert, innerhalb deren alle bzw. ein genau zu definierender Anteil der Orte erreicht werden müssen (Seip, Bill et al.). Falls die bestehende Zahl oder Verteilung der Notarztstandorte die geforderte Hilfsfrist nicht gewährleistet, muss eine Strategie gewählt werden, wie der gesetzlichen Vorgabe genüge getan wird. Die erste Strategie ist der Bau neuer Notarztstandorte unter der Bedingung, dass die bestehenden Standorte unverändert bestehen bleiben. Dies impliziert Bau-, Anschaffungs-, Personal- und weitere Betriebskosten für die neuen Notarztstandorte. Aus betriebswirtschaftlicher Sicht ist hier eine Standortplanung vorzunehmen, damit mit einer möglichst geringen Anzahl von neuen Standorten die bisher unterversorgten Gebiete erreicht werden können.

Als zweite Alternative bietet sich die Verlegung bestehender Standorte an, so dass die bislang unterversorgten Gebiete näher am neuen Standort sind. Dies würde jedoch voraussetzen, dass durch die Verlegung keine neuen „weissen Flecken“ entstehen, die dann zu weit vom neuen Standort entfernt liegen. Dies muss im Einzelfall geprüft werden. Die Verlegung impliziert meist einen Neubau der Rettungswache, d.h. Investitionskosten.

Die dritte Alternative ist der so genannte „Telenotarzt“. Diese innovative Methodik erfordert den Aufbau einer Telenotarztleitstelle an einem zentralen Ort, z.B. einem Maximalversorger in der Region (Rashid, Eder et al. 2015). Sie ist im Einsatzfall in Echtzeit mit den Rettungswagen verbunden und koordiniert den Einsatz (Nowakowski und Fischer 2015). Der Notarzt aus dem benachbarten Gebiet kommt trotzdem, aber bis zu seinem Eintreffen übernimmt der Telenotarzt (ab Eintreffen Rettungswagen) die medizinische Betreuung. Dadurch ist eine qualifizierte notärztliche Betreuung bereits deutlich früher möglich. Nach Eintreffen des Notarztes aus dem Nachbargebiet entscheidet dieser, ob die notärztliche Betreuung während des Transports ins Krankenhaus im Rettungswagen durch 
den Telenotarzt stattfinden kann, oder ob er selbst diesen Transport begleiten muss (Schneiders, Herbst et al. 2012).

Der Telenotarzt wurde in verschiedenen Pilotstudien evaluiert. Aus medizinscher Perspektive sind Verlässlichkeit, Schnelligkeit und Handhabbarkeit sehr positiv zu bewerten, d.h., der Telenotarzt kann einen wichtigen Beitrag zur Abdeckung unzureichend versorgter Gebiete leisten (Bergrath, Reich et al. 2012, Bergrath, Czaplik et al. 2013, Felzen, Brokmann et al. 2016). Auch die technische Zuverlässigkeit (z.B. Netzabdeckung) wurde als ausreichend evaluiert (Bergrath, Rörtgen et al. 2011, Czaplik, Bergrath et al. 2014). Es liegt jedoch keine ökonomische Bewertung vor.

Schliesslich bleibt als vierte Alternative die notärztliche Versorgung der Gebiete, die nicht innerhalb der Hilfsfrist erreicht werden können, durch den Rettungshubschrauber (Hofer und Voelckel 2014). Sie unterliegt allerdings im Vergleich zur bodengebundenen Alternative einer Abhängigkeit vom Wetter. Je nach Ausstattung des Rettungsmittels, Region und Wetter kann der Hubschrauber unter Umständen überhaupt nicht eingesetzt werden.

Die folgende Analyse verfolgt das Ziel, die Alternativen „Neubau Notarztstandorte“, „Telenotarzt“ und „Luftrettung“ ökonomisch zu bewerten. Sie geht von der Annahme aus, dass keine Notarztstandorte geschlossen und durch andere Methoden (Telenotarzt, Luftrettung) ersetzt werden sollen. Für jeden Notfall ist die Präsenz eines Notarztes vorgesehen. Analysiert werden sollen lediglich die Möglichkeiten, die bislang unzureichend versorgten Gebiete möglichst effizient abzudecken. Hierzu werden im zweiten Abschnitt die Methodik und das Fallbeispiel dargestellt. Es folgenden Ergebnisse sowie eine Diskussion.

\section{Methodik}

\subsection{Fallbeispiel}

Die folgende Analyse basiert auf Daten des Kreises Vorpommern-Greifswald im Bundesland Mecklenburg-Vorpommern (Nordost-Deutschland). Bis zum Jahr 2015 galt zwar eine enge Hilfsfrist, aber bestimmte Gebiete wurden von dieser Regelung ausgenommen und als „Sondergebiete“ definiert, für die die Hilfsfristen nicht galten. Sie hätten nur eingehalten werden können, wenn neue Notarztstandorte gebaut worden wären, die jedoch kaum ausgelastet oder kostendeckend gewesen wären. Das neue Rettungsdienstgesetz (2015) hat diese Sonderregelung abgeschafft und fordert die Einhaltung der Hilfsfrist flächendeckend. Als Hilfsfrist wird hierbei die Zeit zwischen Rettungsmittelalarmierung und Ankunft an einer befahrbaren Strasse definiert. Sie soll im Durchschnitt aller Einsätze 10 Minuten nicht überschreiten. Weiterhin soll die Hilfsfrist von 15 Minuten in $95 \%$ der Fälle (Städte $>20.000$ Einwohner) bzw. in $90 \%$ (ländliches Gebiet) gewährleistet sein. Ein weiteres Kriterium ist, dass das Notarzteinsatzfahrzeug (NEF) maximal 5 Minuten nach dem Rettungswagen (RTW) eintreffen soll.

Diese gesetzliche Vorgabe kann so interpretiert werden, dass das NEF nicht später als 20 Minuten (maximal 15 Minuten für RTW zuzüglich maximal 5 Minuten für NEF) nach Rettungsmittelalarmierung eintreffen darf. Abb. 1 zeigt auf Grundlage von Daten des Jahres 2014, in welchen Postleitzahlgebieten der Notarzt maximal 20 Minuten nach der Rettungsmittelalarmierung eingetroffen ist. Es wird deutlich, dass in sechs Postleitzahlregionen mehr als $10 \%$ der NEF-Einsätze die Hilfsfrist für den Notarzt nicht einhalten konnten. 
Eine strengere Interpretation der gesetzlichen Vorgabe führt zu der Forderung, dass der Notarzt maximal fünf Minuten später als der rechtzeitig angekommene RTW eintreffen darf. Abb. 2 zeigt auf Grundlage von Daten des Jahres 2014, in welchen Postleitzahlgebieten der Notarzt maximal 5 Minuten nach dem RTW eingetroffen ist. Es wird deutlich, dass in 14 der 36 Gebiete der Notarzt diese Auflage nicht erfüllt.

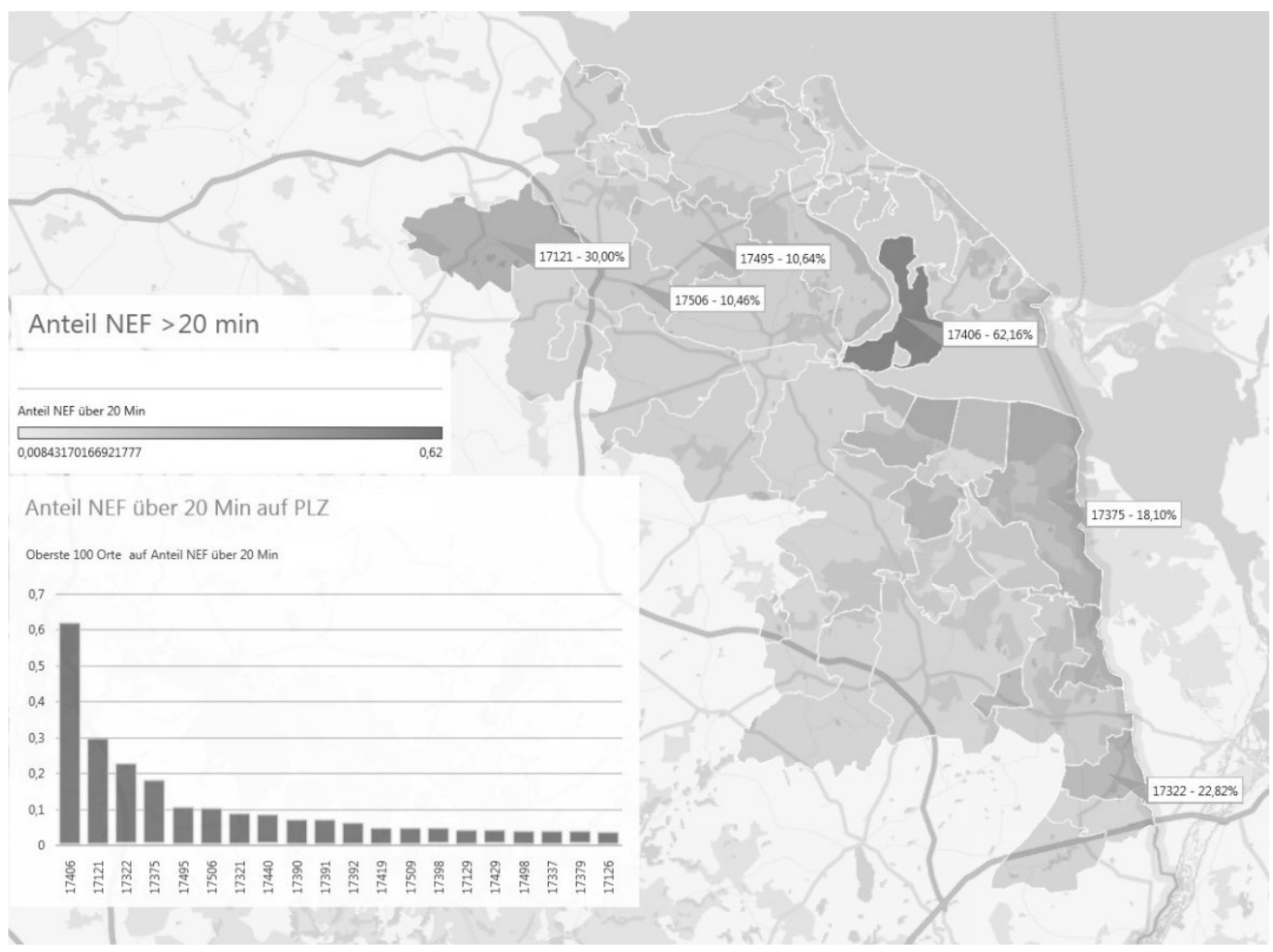

Abb. 1 Anteil der NEF-Einsätze ausserhalb der 20-Minuten Frist nach Rettungsmittelalarmierung

Quelle: Eigene Darstellung nach Rettungsdienst Vorpommern-Greifswald, erstellt mit Microsoft Power Map 


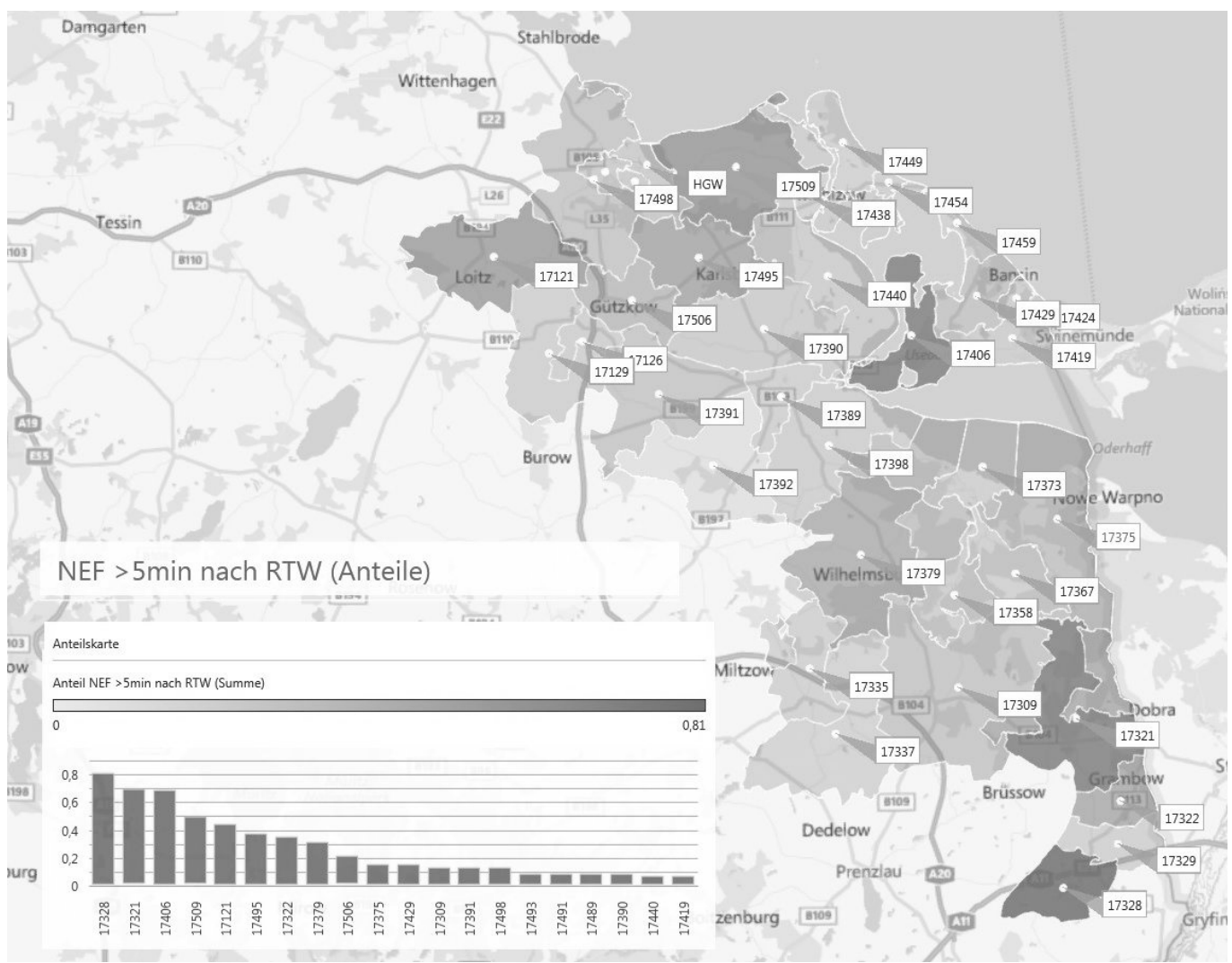

Abb. 2 Anteil der NEF-Einsätze ausserhalb der 5-Minuten Frist nach Eintreffen des RTW Quelle: Eigene Darstellung nach Rettungsdienst Vorpommern-Greifswald, erstellt mit Microsoft Power Map

\section{2. Ökonomische Analyse}

Die ökonomische Analyse entwickelt Kostenfunktionen für die drei Alternativen (1) Bau neuer Notarztstandorte, (2) Telenotarzt und (3) Luftrettung. Zur Veranschaulichung werden in $A b b .3$ und $A b b .4$ die Alternativen noch einmal mit ihren möglichen Einsätzen dargestellt. Abb. 4 (links) zeigt den Fall, dass der Telenotarzt bereits mit dem Rettungswagen am Notfallort in Verbindung steht, bis der Notarzt aus dem angrenzenden Gebiet ankommt. Dieser kann dann entweder zurück an seinen Standort fahren oder den Patienten begleiten. 


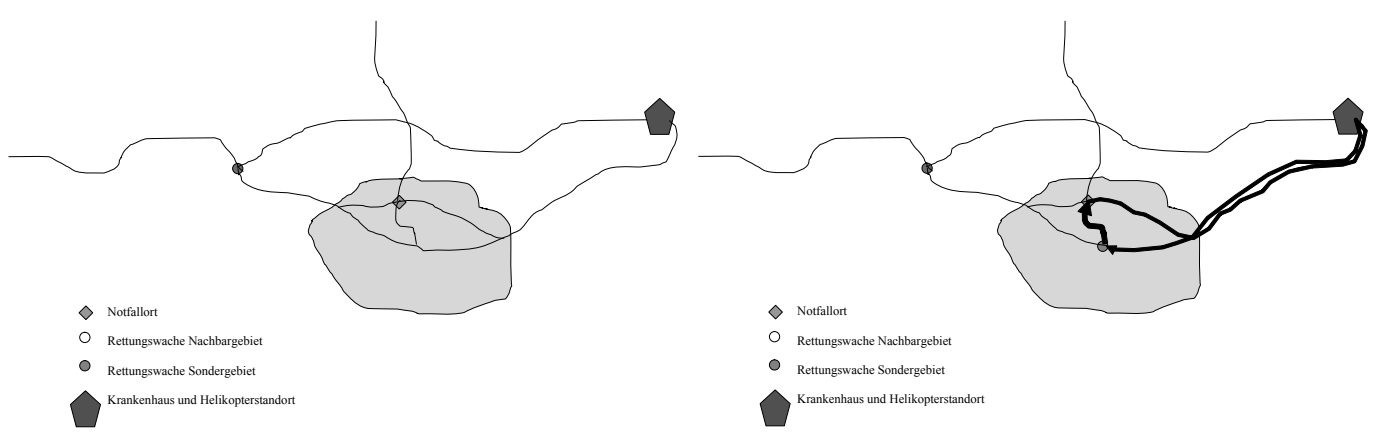

Abb. 3 Skizze, Ausgangslage und neuer Notarztstandort

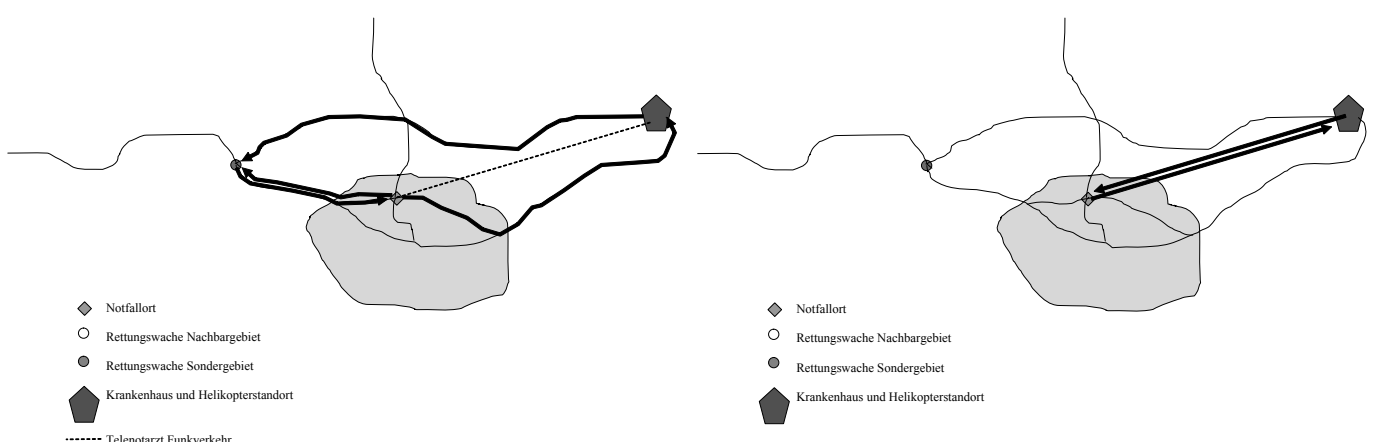

Abb. 4 Skizze, Telenotarzt und Luftrettung

Jeder dieser drei Alternativen ist kostenmässig zu bewerten und mit einer Break-Even-Analyse zu vergleichen. Hierzu werden folgende Variablen und Konstanten definiert:

$\mathrm{K}_{\mathrm{I}} \quad$ Kosten für Alternative „Ausbau Notarztstandorte“ [€ p.a.]

$\mathrm{K}_{\mathrm{II}} \quad$ Kosten für Alternative „Telenotarzt“ [€ p.a.]

$\mathrm{K}_{\mathrm{III}} \quad$ Kosten für Alternative „Luftrettung“ [ $€$ p.a.]

x Anzahl der zusätzlichen Notarztstandorte bzw. der Gebiete, die bislang nicht innerhalb der Hilfsfrist erreicht werden

$\mathrm{KA}_{\mathrm{TN}} \quad$ Kapazität von Fahrzeugen, die ein Telenotarzt maximal gleichzeitig handeln kann

$\mathrm{KA}_{\mathrm{h}} \quad$ Kapazität des Hubschraubers, d.h. maximale Zahl von Einsätzen pro Jahr

$\mathrm{E}_{0 \mathrm{~h}} \quad$ Zahl der derzeitigen Hubschraubereinsätze Einsätze pro Jahr

e Einsatzhäufigkeit in einem Gebiet, das bislang nicht innerhalb der Hilfsfrist erreicht werden kann [Einsätze pro Jahr]

V Zahl der Verlegungen pro Jahr insgesamt

Y Zahl der Einsätze in allen bisherigen Gebieten [Einsätze pro Jahr]

d Durchschnittliche Zeit pro Verlegung (hin- und zurück) [h]

w Zahl der Notarztstandorte derzeit 
$\mathrm{c}_{\mathrm{I}} \quad$ Jährliche Fixkosten pro Notarztstandort (Betriebs- und anteilige Investitionskosten) [€ p.a.]

$\mathrm{c}_{\mathrm{II}} \quad$ Jährliche Kosten pro Telemedizinarbeitsplatz (Betriebs- und anteilige Investitionskosten) [€ p.a.]

c IIf Jährliche Kosten pro Telenotarztfahrzeug (Abschreibung technische Umrüstung) [€ p.a.]

C IIIa Jährliche Fixkosten pro Hubschrauber in der Grundausstattung (fixe Betriebs- und anteilige Investitionskosten) [€ p.a.]

c IIIn Jährliche Fixkosten pro Hubschrauber für 18-Stunden Tag und Nachtflugeignung (fixe Betriebs- und anteilige Investitionskosten) [€ p.a.]

$c_{h} \quad$ Variable Kosten pro Hubschraubereinsatz

$c_{e} \quad$ Durchschnittliche variable Kosten eines Einsatzes in einem Sondergebiet [€ pro Einsatz]

$c_{\mathrm{a}} \quad$ Durchschnittliche variable Anrückkosten für ein Fahrzeug ausserhalb eines Sondergebietes bis zum Unfallort im Sondergebiet [€ pro Einsatz]

$\mathrm{c}_{\mathrm{s}} \quad$ Durchschnittliche variable Kosten pro Notarzteinsatz, wenn der Arzt von ausserhalb des Sondergebietes anrücken muss [€ pro Einsatz]

r

Anteil von Einsätzen, die zusätzlich zum Telenotarzt noch einen bodengebundenen Arzt von ausserhalb des Sondergebietes benötigen

Der Parameter $\mathrm{x}$ wird als parametrische Konstante definiert und im Rahmen der BreakEven-Analyse variiert, d.h., es wird die Anzahl von Gebieten gesucht, bis zu der bzw. ab der sich eine bestimmte Alternative als dominant erweist. Zur Vereinfachung wird hier der Begriff „Sondergebiet“ verwendet, er darf jedoch nicht mit der rechtlichen Definition gleichgesetzt werden. Vielmehr handelt es sich um all diejenigen Gebiete, die bislang nicht innerhalb der Hilfsfrist versorgt werden, auch wenn dies im Durchschnitt der Einsätze eines Notarztstandortes untergeht.

Die Kosten für Alternative „Ausbau Notarztstandorte“ setzen sich zusammen aus den Investitionskosten für die Standorte, den Kosten des Hubschraubers, wenn der Notarzt in einer Verlegung gebunden ist, sowie den variablen Kosten für die Notarzteinsätze in einem Sondergebiet. Die variablen Kosten berechnen sich als das Produkt der Zahl der zusätzlichen Notfallstandorte, der Einsatzhäufigkeit in einem Sondergebiet und der durchschnittlichen variablen Kosten, die so ein Einsatz kostet (inkl. der Betreuung während des Transports zum Krankenhaus).

Wenn ein Notarzt durch eine Verlegung gebunden ist, wird alternativ für die Notfallversorgung der Hubschrauber verwendet. V•d ist die benötigte Zeit für alle Verlegungen, 365 - 24 • $(\mathrm{x}+\mathrm{w})$ die zur Verfügung stehende Zeit der Notärzte in allen Notarztstandorten. Damit ist $\frac{\mathrm{v} \cdot \mathrm{d}}{365 \cdot 24 \cdot(\mathrm{x}+\mathrm{w})}$ die Wahrscheinlichkeit, dass ein Hubschrauber für einen Notfall gerufen werden muss, weil der Notarzt in einer Verlegung gebunden ist. $\mathrm{Da}(\mathrm{Y}+\mathrm{x} \bullet \mathrm{e})$ die Zahl maximal möglicher Hubschraubereinsätze ist, entspricht $\frac{\mathrm{v} \bullet \mathrm{d}}{365 \cdot 24 \bullet(\mathrm{x}+\mathrm{w})} \bullet(\mathrm{Y}+\mathrm{x} \bullet \mathrm{e})$ der Zahl der Hubschraubereinsätze, die geflogen werden müssen, weil ein bodengebundener Notarzt in einem Verlegetransport gebunden ist. Dieses Produkt muss noch mit den variablen Kosten eines Hubschraubereinsatzes $c_{h}$ gewichtet werden. Damit ergeben sich 
für den realistischen Fall, dass die Kosten für Alternative (1) „Ausbau Notarztstandorte“ als

$\mathrm{K}_{I}=c_{I} \cdot x+c_{h} \cdot \frac{V \cdot d}{365 \cdot 24 \cdot(x+w)} \cdot(Y+x \cdot e)+x \cdot e \cdot c_{e}$

Die Kosten für den Telenotarzt setzen sich zusammen aus den Investitionskosten für den Telenotarztarbeitsplatz (wobei sprungfixe Kosten zu berücksichtigen sind, da in Abhängigkeit von der Zahl der zu betreuenden Gebiete die Zahl der Plätze steigen muss), den jährlichen Fixkosten pro Telenotarztfahrzeug und den Kosten für von anderen Gebieten zugezogenen bodengebundenen Notärzten. Letztere müssen unterschieden werden in Kosten für den Fall, dass der Notarzt aus dem anderen Gebiet nur bis zum Unfallort fährt und dann die Betreuung bis zum Krankenhaus dem Telenotarzt überlässt, und den Kosten, falls er auch noch bis zum Krankenhaus fährt (Wahrscheinlichkeit $\mathrm{r} \%$ ). Kosten für den Hubschrauber für Verlegungen, die den bodengebundenen Notarzt binden, fallen in dieser Alternative nicht an, da der Telenotarzt den Transport überwachen kann. Damit sind die Kosten für Alternative (2) „Telenotarzt“ gegeben als:

$\mathrm{K}_{I I}=c_{I I} \cdot \operatorname{trunc}\left(1+\frac{x}{K A_{T N}}\right)+c_{I I f} \cdot x+r \cdot x \cdot e \cdot c_{s}+(1-r) \cdot x \cdot e \cdot c_{a}$

Die Kosten für die Luftrettung für die Versorgung dieser Standorte sind die Summe aus fixen und variablen Kosten. Ein Teil der Fixkosten $\left(c_{\text {III }}\right)$ deckt die Grundausstattung des Hubschraubers ab, so wie der derzeit existiert. Sie fallen erst zusätzlich an, wenn die Kapazitätsgrenze überschritten würde. Ein weiterer Teil der Fixkosten umfasst den Ausbau auf einen 18-Stunden Tag sowie den zweiten Piloten. Dieser Fixkostenteil fällt bereits von Anfang an an. Damit errechnen sich die Kosten für Alternative „Luftrettung“ als:

$\mathrm{K}_{I I I}=c_{I I I a} \bullet \operatorname{trunc}\left(\frac{e \cdot x+E_{0 h}}{K A_{h}}\right)+c_{I I I n} \bullet \operatorname{trunc}\left(1+\frac{e \cdot x+E_{0 h}}{K A_{h}}\right)+c_{h} \cdot x \cdot e$

\subsection{Daten}

Tab. 1 gibt die Grunddaten eines Notarztstandortes wieder. Damit ergeben sich Fixkosten von $613.500 €$, Fahrkosten für den Einsatz im eigenen Gebiet von durchschnittlich 16,80 €, Fahrkosten, falls der Notfall vollständig von einem Notarzt ausserhalb des Sondergebietes versorgt wird, von $28,00 €$ und von 11,20 €, falls der Notarzt von ausserhalb des Sondergebietes kommt, jedoch die weitere Betreuung während des Transports dem Telenotarzt überlässt.

\section{Tab. 1 Grunddaten Notarztstandort}

Parameter

Wert

Bau einer Notarztwache nach DIN-Norm $350.000,00 €$

Abschreibung Bauten [Jahre]

Investition an Wacheninventar

$20.000,00 €$

Abschreibung Inventar [Jahre]

Laufende Gebäudenebenkosten (Reinigung, Versicherung, Wasser, Strom, Abfall, Wartung, etc.): 
Personalkosten Notarzt pro Jahr bei 365 Tage mit je 24 Stunden (Standort nicht an einem Krankenhaus):

Rettungsassistent/Notfallsanitäter als Fahrer des Notarzteinsatzfahrzeuges: ca. 4,6 VK bei 365 Tage 24h Betrieb

$190.000,00 €$

Anschaffung Notarzteinsatzfahrzeug (Afa 5 Jahre, ohne Medizintechnik):

$65.000,00 €$

Abschreibung Fahrzeug [Jahre]

Anschaffung Medizintechnik (Afa 8 Jahre) für Notarzteinsatzfahrzeug:

$40.000,00 €$

Abschreibung Medizintechnik [Jahre]

Verwaltungskostenaufwand (kalkulatorisch ca.5\% der Personalkosten):

$26.500,00 €$

Fahrkosten pro km

durchschnittliche Strecke im eigenen Gebiet einfach [km]

Tab. 2 zeigt die Grunddaten des Telenotarztes. Hierbei entstehen Fixkosten der Zentrale von 1.051.200,00 € p.a. sowie Fixkosten pro Notarztfahrzeug in Höhe von 10.500,00€ p.a.

\section{Tab. 2 Grunddaten Telenotarzt}

Parameter

Wert

Investition Hardware pro Rettungswagen

$42.000,00 €$

Abschreibungsperiode Hardware pro Rettungswagen

Investitionskosten Netzwerk

49.200,00€

Abschreibungsperiode Netzwerk

Investitionskosten Telenotarztarbeitsplatz

$48.000,00 €$

Abschreibungsperiode Telenotarztarbeitsplatz

Technischer Betrieb pro Jahr (Simkarten, Lizenzen, Telenotarztzentrale)

$180.000,00 €$

Support, Wartung, Assurance

$154.700,00 €$

Ausbildung, Supervision QM

$90.000,00 €$

Ausfallsicherheit und Desaster Recovery

$74.200,00 €$

Personalkosten bei 365 Tagen mit ja 24 Stunden

$528.000,00 €$

Tab. 3 gibt die Grunddaten der Luftrettung wieder. Vorausgesetzt ist hierbei, dass die Luftrettung auf 18 Stunden pro Tag ausgebaut wird. Dies erfordert zusätzliches Personal mit entsprechenden Kosten. Die Fixkosten belaufen sich damit auf 1.480.000,00 €, die variablen Kosten pro Hubschraubereinsatz auf 1.180,00€. Dies entspricht Minutenkosten von $52,60 €$. 
Tab. 3 Grunddaten Luftrettung

Parameter

Wert

Investitionskosten Hangar

$1.000 .000,00 €$

Abschreibungsperiode Hangar

Investitionskosten sonstige Bodentechnik

$400.000,00 €$

Abschreibungsperiode Bodentechnik

Investitionskosten Hubschrauber Grundausstattung

$4.000 .000,00 €$

Abschreibungsperiode Hubschrauber Grundausstattung

Investitionskosten Nachtflugtechnik

$200.000,00 €$

Abschreibungsperiode Nachtflugtechnik

Zahl der Piloten bisher (12 Stunden, 365 Tage)

Zahl der Piloten für Ausbau (18 Stunden, 365 Tage)

Sonstiges Personal

Kosten Pilot p.a.

Kosten sonstiges Personal p.a.

Wartung p.a.

Sonstige Betriebskosten p.a.

Durchschnittliche Flugstrecke [km]

Benzinkosten pro km

Medizinisches Personal pro Flug

Tab. 4 fasst diese und weitere Parameter zusammen.

Tab. 4 Modellkonstanten

Konstante

Wert

Kapazität des Hubschraubers, d.h. maximal Zahl von Einsätzen pro Jahr 1600

Anzahl derzeitiger Hubschraubereinsätze pro Jahr

Einsatzhäufigkeit in einem Sondergebiet pro Jahr [Einsätze pro Jahr]

Zahl der notarztbegleiteten Verlegungen pro Jahr insgesamt in Kreis

Durchschnittliche Zeit pro Verlegung (hin- und zurück) [h]

1,92

Zahl der Notarztstandorte derzeit

jährliche Fixkosten pro Notarztstandort

$613.500,00 €$

jährliche Kosten pro Telemedizinarbeitsplatz

Jährliche Fixkosten pro Telenotarztfahrzeug (Abschreibung technische Um-

$1.051 .200,00 €$ rüstung)

jährliche Fixkosten pro Hubschrauber in der Grundausstattung

jährliche zusätzliche Fixkosten für Nachtflug und 18-Stunden Tag

variable Kosten pro Hubschraubereinsatz

$10.500,00 €$

Durchschnittliche variable Kosten pro Einsatz in einem Sondergebiet

$1.120 .000,00 €$

$360.000,00 €$

$1.180,00 €$

$16,80 €$

Durchschnittliche variable Anrückkosten für ein Fahrzeug ausserhalb eines

Sondergebietes bis zum Unfallort im Sondergebiet

$11,20 €$ 
durchschnittliche variable Kosten pro Notarzteinsatz, wenn der Arzt von

$28,00 €$ ausserhalb des Sondergebietes anrücken muss

Anteil der Einsätze, die zusätzlich zum Telenotarzt noch eines fahrenden

Notarztes bedürfen

Zahl der Einsätze in bisherigen Gebieten [Einsätze pro Jahr]

Zahl von Rettungsgebieten, die ein Telenotarzt handeln kann

Kapazität von Fahrzeugen, die ein Telenotarzt maximal gleichzeitig handeln kann

Relevant ist auch die Schätzung der Zahl der Einsätze, die ein Telenotarzt parallel handeln kann. Mathematisch handelt es sich um ein $\mathrm{M} / \mathrm{M} / \mathrm{c}$ Warteschlagensystem, d.h., unter der Bedingung Poisson-verteilter Ankünfte und exponentiell-verteilter Abfertigungen ergibt sich die Wahrscheinlichkeit, dass ein Notfall nicht gleich versorgt werden kann, als

$$
C\left(c, \frac{\lambda}{\mu}\right)=\frac{\left(\frac{(c \rho)^{c}}{c !}\right)\left(\frac{1}{1-\rho}\right)}{\sum_{k=0}^{c-1} \frac{(c \rho)^{k}}{k !}+\left(\frac{(c \rho)^{c}}{c !}\right)\left(\frac{1}{1-\rho}\right)}
$$

mit

c Zahl der parallel möglichen notärztlichen Betreuungen

$\rho \quad$ Verkehrsdichte mit $\rho=\frac{1}{c \cdot \mu}$

1 Ankunftsrate [Notarztfälle pro Stunde]

$\mu$ Abfertigungsrate [Notarztfälle pro Stunde]

Unter Annahmen von Tab. 4 ergibt sich die Wahrscheinlichkeit, dass mehr als c Notfälle gleichzeitig bearbeitet werden müssen, als 4,87 \% falls c=1 (nur ein Fall gleichzeitig möglich) und $0,1 \%$ falls $c=2$. Folglich ist die Wahrscheinlichkeit, dass die Kapazität des Telenotarztes überschritten wird, in den Gebieten, die bislang die Hilfsfrist nicht einhalten, sehr gering. Zur Vereinfachung wurde damit $K A_{\mathrm{TN}}=13 \mathrm{c}$ geschätzt. Diese Schätzung ist als konservativ zu bezeichnen.

\section{Ergebnisse}

Abb. 5 zeigt die Kostensituation für die drei Alternativen auf Grundlage der Parameter von Tab. 4. Es wird deutlich, dass der Telenotarzt kostengünstiger als der Ausbau neuer Notarztstandorte ist, wenn mindestens zwei neue Notarztstandorte errichtet werden müssten, um die bislang nicht ausreichend innerhalb der notärztlichen Hilfsfrist erreichten Gebiete zu versorgen. Wenn weniger als sieben Gebiete bislang nicht innerhalb der Hilfsfristen erreicht wurden, ist die Verwendung des Hubschraubers hingegen kostengünstiger. Der Kostensprung bei der Luftrettung zwischen $\mathrm{x}=6$ und $\mathrm{x}=7$ weist auf die Kapazitätsgrenze des Hubschraubers hin, d.h., an dieser Stelle müsste ein weiteres Gerät angeschafft werden. 


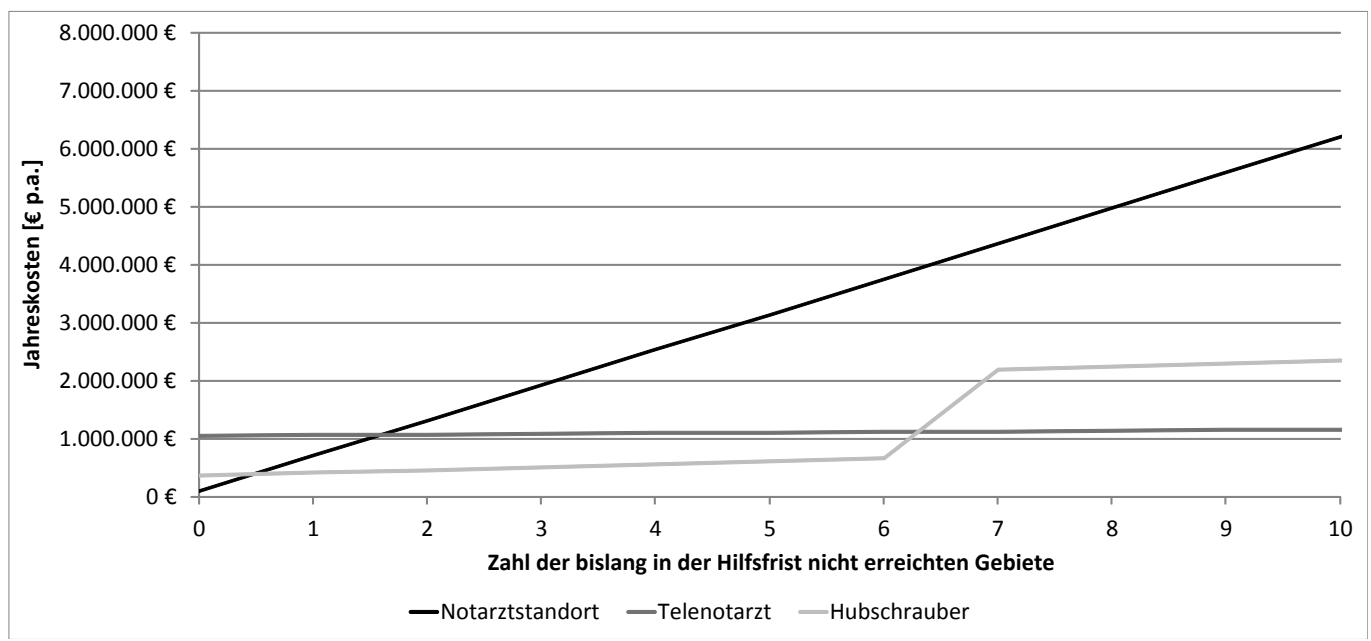

Abb. 5 Break-Even-Analyse

Abb. 6 zeigt exemplarisch für sechs bislang nicht ausreichend versorgte Gebiete die Kostenanteile. Es wird deutlich, dass die Fixkosten extrem dominant sind. Dies liegt primär daran, dass in diesen dünn besiedelten Gebieten die Einsatzhäufigkeit sehr gering ist, so dass vor allem Vorhaltekosten anfallen.

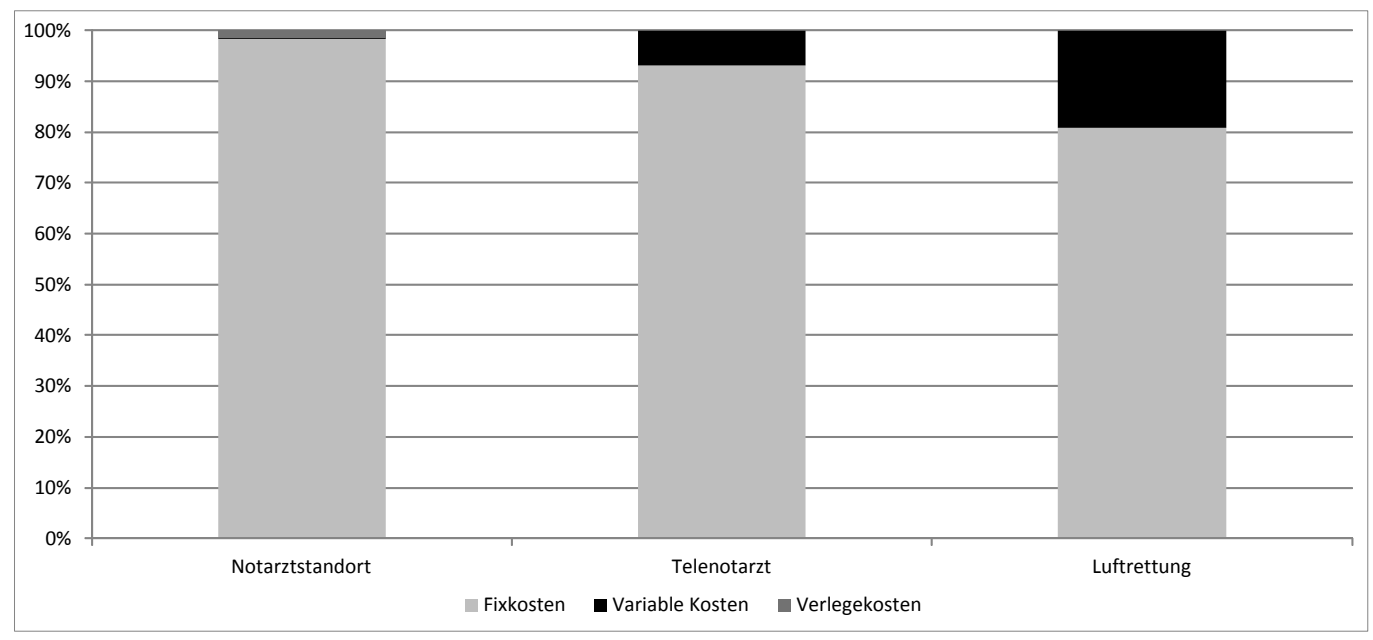

Abb. 6 Kostenanteile (für $\mathrm{x}=6$ )

Die Ergebnisse sind erstaunlich stabil für Parameteränderungen. Hierbei wurden die jeweiligen Parameter univariat solange verändert, bis sich eine andere Alternative gegenüber dem Telenotarzt als dominant erweist. Tatsächlich erscheint der Ausbau von Notarztstandorten gegenüber dem Telenotarzt nur dann sensitiv, wenn die Zeit, die für eine Verlegung benötigt wird, über 8 Stunden steigt, was völlig unrealistisch ist. In diesem Fall ist der Telenotarzt bereits ab einem zusätzlichen Gebiet $(\mathrm{x}=1)$ dominant. Ansonsten bestäti- 
gen alle univariaten Parameterveränderungen die Aussage, dass der Telenotarzt gegenüber dem Ausbau der Notarztstandorte dominant ist.

Auch die Entscheidung, dass der Ausbau der Luftrettung gegenüber dem Telenotarzt für $\mathrm{x}<7$ dominant ist, ist sehr stabil. Das Modell geht davon aus, dass die Erweiterung auf Nachtflug zwei statt einem Piloten erfordern würde, so dass insgesamt für den 18-Stunden Tag sechs Piloten angestellt sein müssten, um die Ganzjahrespräsenz zu garantieren. Die Auflösung dieser Annahme verändert jedoch den Sprung bei x=6 nicht. Relevant wäre lediglich, wenn die Fixkosten des Hubschraubers bereits vollständig auf das erste zu versorgende Gebiet angerechnet würden, d.h., wenn für die Versorgung dieser Gebiete von Anfang an ein zusätzlicher Hubschrauber angeschafft werden müsste. In diesem Fall wäre der Telenotarzt bereits bei $\mathrm{x}=2$ dominant. Allerdings ist dies nicht realistisch.

\section{Diskussion}

Die vorliegenden Analysen zeigen auf, dass mit gegebenen Kostenschätzungen der Telenotarzt gegenüber dem Aufbau von neuen Notarztstandorten die vorzuziehende Alternative für die notärztliche Versorgung der Bevölkerung in denjenigen Gebieten ist, in denen bislang die Hilfsfristen nicht eingehalten werden können. Die Ergebnisse sind eindeutig und stabil für den Fall, dass mindestens zwei solcher Gebiete ausgewiesen werden können. Wie $A b b .1$ und $A b b .2$ zeigt, ist dies für den Kreis Vorpommern-Greifswald der Fall. Im Vergleich zum Rettungshubschrauber ist der Telenotarzt effizient, wenn mehr als sechs derartige Gebiete vorliegen. Hierbei ist zu bedenken, dass die Wahrscheinlichkeit, dass der Rettungshubschrauber bei Alarmierung belegt ist, mit zunehmender Zahl der zusätzlich zu versorgenden Gebieten steigt. Wenn man das Risiko einer unzureichenden Kapazität des Rettungshubschraubers einbezieht, dürfte deshalb bereits bei fünf zusätzlich zu versorgenden Gebieten der Telenotarzt dominant sein.

Es zeigt sich folglich, dass der Telenotarzt nicht nur auf medizinischer Sicht (Nowakowski und Fischer 2015, Rashid, Eder et al. 2015), sondern auch aus ökonomischer Perspektive eine zukunftsfähige Innovation darstellt. Für städtische Regionen ist dies wenig erstaunlich (Wielpütz, Skorning et al. 2010), aber die vorliegenden Analysen zeigen dies auch für Flächenländern mit dünner Bevölkerungsdichte wie Nordost-Deutschland oder Teile der Schweiz auf. Verschärft wird die Situation durch natürliche Hindernisse, die die Anfahrtswege zum Teil erheblich verlängern und damit eine fristgerechte Rettung fast unmöglich machen. In Vorpommern sind dies insbesondere Flüsse, Seen, Küstenlinien (inkl. periodisch für den Schiffsverkehr zu öffnender Brücken) und Sumpfgebiete, während in der Schweiz vor allem an Seen und Berge zu denken ist. Diese natürlichen Barrieren erfordern auch eine Standortplanung, die nicht von euklidischen Distanzen, sondern vom tatsächlichen Wegenetz ausgeht. Hinzu kommt noch die saisonal schwankende Zugänglichkeit und Verwendbarkeit der Rettungsmittel. In Vorpommern sind beispielsweise beim Bettenwechsel auf den Urlaubsinseln Usedom und Rügen sowie bei den Herbst- und Winterstürmen die Rettungsfristen kaum zu halten, insbesondere da bei Stürmen und in der Dunkelheit (die im Winter bis zu 18 Stunden pro Tag ausmacht) der Hubschrauber nur bedingt einsetzbar ist. In der Schweiz dürften Schneefälle und Stürme ebenso die Anfahrtszeiten erheblich erhöhen und gleichzeitig den Einsatz des Rettungshubschraubers einschränken. Diese Argumente stärken die Forderung nach dem baldigen Einsatz des Telenotarztes. 
Die Analyse setzt voraus, dass die Qualität der telenotärztlichen Betreuung sowie der Betreuung durch bodengestützte Notarztversorgung gleich ist. Im Rahmen dieses Artikels konnte nicht untersucht werden, ob beispielsweise die Netzabdeckung in ländlichen Regionen der Schweiz ausreicht. Weiterhin werden Interdependenzen zwischen den obigen Parametern nicht berücksichtigt. Beispielsweise kann die Zahl der Notarzteinsätze mit der Existenz eines Notarztstandortes steigen, da die Bevölkerung den Standort kennt und deshalb im Zweifelsfall eher den Notarzt anfordert. Diese - unwahrscheinlichen, aber nicht auszuschliessenden - verhaltensbedingten Interdependenzen der Parameter konnten im vorliegenden Ansatz nicht berücksichtigt werden.

Aus ökonomischer Sicht ist folglich die Umsetzung des Telenotarztes in Vorpommern und in ländlichen Regionen der Schweiz zu empfehlen. Ex-Post sollte die Validität der obigen Parameter ebenso evaluiert werden wie die medizinischen Outcomes und die Zufriedenheit der Patienten.

\section{Literatur}

Bergrath, S., M. Czaplik, R. Rossaint, F. Hirsch, S. K. Beckers, B. Valentin, D. Wielpütz, M.-T. Schneiders und J. C. Brokmann (2013). „Implementation phase of a multicentre prehospital telemedicine system to support paramedics: feasibility and possible limitations." Scand J Trauma Resusc Emerg Med 21(54): 1-10.

Bergrath, S., A. Reich, R. Rossaint, D. Rörtgen, J. Gerber, H. Fischermann, S. K. Beckers, J. C. Brokmann, J. B. Schulz und C. Leber (2012). „Feasibility of prehospital teleconsultation in acute stroke-a pilot study in clinical routine. " PloS one 7(5): e36796.

Bergrath, S., D. Rörtgen, R. Rossaint, S. K. Beckers, H. Fischermann, J. C. Brokmann, M. Czaplik, M. Felzen, M.-T. Schneiders und M. Skorning (2011). „Technical and organisational feasibility of a multifunctional telemedicine system in an emergency medical service-an observational study." Journal of telemedicine and telecare 17(7): 371-377.

Czaplik, M., S. Bergrath, R. Rossaint, S. Thelen, T. Brodziak, B. Valentin, F. Hirsch, S. Beckers und J. Brokmann (2014). „Employment of telemedicine in emergency medicine.“ methods of information in medicine 53(2): 99-107.

Dünkel, F., M. Herbst und T. Schlegel (2014). Think Rural!: Dynamiken des Wandels in peripheren ländlichen Räumen und ihre Implikationen für die Daseinsvorsorge, Springer-Verlag.

Felzen, M., J. C. Brokmann, S. K. Beckers, M. Czaplik, F. Hirsch, M. Tamm, R. Rossaint und S. Bergrath (2016). „Improved technical performance of a multifunctional prehospital telemedicine system between the research phase and the routine use phase-an observational study. "Journal of telemedicine and telecare: 1357633 X16644115.

Fleßa, S. (2016). „Verantwortung teilen - gemeinsam planen. Die Rolle der primärversorgenden Ärzte im Regional Health Care. "Zeitschrift für Allgemeinmedizin 91: 499-505.

Fleßa, S. und V. Gieseler (2016). Die Rolle der Krankenhäuser im ländlichen Raum. Daseinsvorsorge und Gemeinwesen im ländlichen Raum. M. Herbst, F. Dünkel und B. Stahl. Wiesbaden, Springer: 43-60.

Herbst, M., F. Dünkel und B. Stahl (2016). Daseinsvorsorge und Gemeinwesen im ländlichen Raum. Wiesbaden, Springer.

Hofer, G. und W. Voelckel (2014). „Stellenwert der Hubschrauberrettung. “ Medizinische Klinik-Intensivmedizin und Notfallmedizin 109(2): 95-99. 
Nowakowski, N. und F. Fischer (2015). „Telematikanwendungen in der präklinischen Notfallmedizin in Deutschland-Einsatzmöglichkeiten und Herausforderungen. " Der Notarzt 31(04): 177-183.

Rashid, A., P. A. Eder und U. Kippnich (2015). „Telemedizin im Rettungsdienst-Möglichkeiten für den Einsatzalltag." retten! 4(04): 256-260.

Schneiders, M.-T., S. Herbst, D. Schilberg, I. Isenhardt, S. Jeschke, H. Fischermann, S. Bergrath, R. Rossaint und M. Skorning (2012). „Telenotarzt auf dem Prüfstand.“ Notfall+ Rettungsmedizin 15(5): 410-415.

Seip, C., R. Bill und A. Kinskofer. „Notfallversorgung der Bevölkerung in Mecklenburg-Vorpommern." Notfall+ Rettungsmedizin: 1-9.

Wielpütz, D., M. Skorning und R. Rossaint (2010). Telemedizin im Rettungsdienst: Das Aachener Projekt Med-on-@ ix. Stand der Evaluationsphase. Akzeptanz, Nutzungsbarrieren und ethische Implikationen neuer Medizintechnologien. Die Anwendungsfelder Telemedizin und Inkorporierte Technik. D. Groß. Kassel, Kassel University Press. 8: 17-20.

Steffen Fleßa, Professor für Gesundheitsmanagement an der Universität Greifswald, Schwerpunkte Versorgung im ländlichen Raum, quantitative Methoden, Krankenhausmanagement; Mitinitiator des Forschungskonsortiums „Think Rural“.

Anschrift: Universität Greifswald, Lehrstuhl für Allgemeine Betriebswirtschaftslehre und Gesundheitsmanagement, Friedrich-Loeffler-Straße 70, D-17487 Greifswald, Tel.: 03834-862476, Fax: 03834-862475,E-Mail: steffen.flessa@uni-greifswald.de

Markus Krohn, ist Mitarbeiter am Lehrstuhl von Steffen Fleßa, beschäftigt sich unter anderem mit der Wirtschaftlichkeit des Rettungsdienstes.

Anschrift: Universität Greifswald, Lehrstuhl für Allgemeine Betriebswirtschaftslehre und Gesundheitsmanagement, Friedrich-Loeffler-Straße 70, D-17487 Greifswald, Tel.: 03834-862476, Fax: 03834-862475, E-Mail: markus.krohn@uni-greifswald.de

Dirk Scheer ist Jurist und Sozialdezernent des Kreises Vorpommern-Greifswald, einer der Kreise in Deutschland mit der größten Fläche, geringsten Bevölkerungsdichte und niedrigen Durchschnittseinkommen.

Anschrift: Sozialdezernent, Kreis Vorpommern-Greifswald, Feldstraße 85 a, D-17489 Greifswald, Tel.: 03834 8760-2001, Fax: 03834 8760-92001, E-Mail: Dirk.Scheer@kreisvg.de

Klaus Hahnenkamp ist Leiter der Klinik für Anästhesiologie, Anästhesie, Intensiv-, Notfall- und Schmerzmedizin an der Universitätsmedizin Greifswald, Schwerpunkt Erforschung von Innovationen des Rettungswesens.

Anschrift: Klinik für Anästhesiologie, Anästhesie, Intensiv-, Notfall- und Schmerzmedizin, Universitätsmedizin Greifswald, Ferdinand-Sauerbruch Str., D-17475 Greifswald, Tel.: 0383486 5801, Fax: 0383486 5802, E-Mail: klaus.hahnenkamp@uni-greifswald.de 